(W)

Check for

updates

Cite as

Nano-Micro Lett.

(2022) 14:66

Received: 6 November 2021

Accepted: 13 January 2022

Published online: 23 February 2022

(C) The Author(s) 2022

\section{High-Performance Blue Quasi-2D Perovskite Light-Emitting Diodes via Balanced Carrier Confinement and Transfer}

\author{
Zhenwei Ren ${ }^{1}$, Jiayun $\mathrm{Sun}^{1}$, Jiahao $\mathrm{Yu}^{2}$, Xiangtian Xiao ${ }^{1}$, Zhaojin Wang ${ }^{2}$, Ruijia Zhang ${ }^{1}$, \\ Kai Wang ${ }^{2}$, Rui Chen ${ }^{\circledR}$, Yu Chen ${ }^{3}$, Wallace C. H. Choy ${ }^{1,4} \bowtie$
}

\title{
HIGHLIGHTS
}

- A new concept to optimize the passivating agents for the balance of the carrier confinement and transfer in blue quasi-2D perovskites.

- The moderate tributylphosphine oxide is introduced into the quasi-2D perovskites to simultaneously strengthen the carrier confinement for massive radiative recombination as well as promote efficient carrier transfer in the quasi-2D perovskites.

- High-efficient and stable blue PeLEDs are achieved with an external quantum efficiency of $11.5 \%$ and a very long operational stability of 41.1 min without any shift of the electroluminescence spectra.

ABSTRACT Extensive investigation of the passivating agents has been performed to suppress the perovskite defects. However, very few attentions have been paid to rationally design the passivating agents for the balance of the carrier confinement and transfer in quasi-2D perovskites, which is essential to achieve high-performance perovskite LEDs (PeLEDs). In this work, tributylphosphine oxide (TBPO) with moderate carbon chain length is demonstrated as a decent passivator for the quasi-2D perovskites by strengthening the carrier confinement for massive radiative recombination within the perovskites, and more

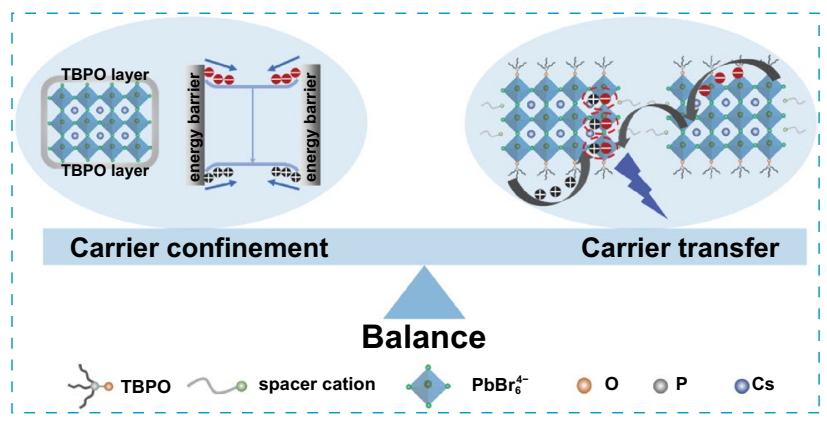
importantly providing efficient carrier transfer in the quasi-2D perovskites. Benefiting from these interesting optoelectronic properties of TBPO-incorporated perovskites, we achieve high-efficient blue PeLEDs with an external quantum efficiency up to $11.5 \%$ and operational stability as long as $41.1 \mathrm{~min}$ without any shift of the electroluminescence spectra. Consequently, this work contributes an effective approach to promote the carrier confinement and transfer for high-performance and stable blue PeLEDs.

KEYWORDS Blue perovskite LEDs; Carrier confinement and transfer; Defect passivation; 2-Dimentional

Kai Wang,wangk@ sustc.edu.cn; Rui Chen, chenr@sustech.edu.cn; Wallace C. H. Choy, chchoy@eee.hku.hk

Department of Electrical and Electronic Engineering, The University of Hong Kong, Pokfulam Road, Hong Kong, People's Republic of China

2 Department of Electrical and Electronic Engineering, Southern University of Science and Technology, Shenzhen 518055, People's Republic of China

3 School of Optoelectronic Science and Engineering, Soochow University, Suzhou 215006, People's Republic of China

4 Guangdong-Hong Kong-Macao Joint Laboratory for Photonic-Thermal-Electrical Energy Materials and Devices, Shenzhen 518055,

People's Republic of China 


\section{Introduction}

The excellent properties of metal halide perovskites, such as high color purity, tunable emission color, high photoluminescence quantum yields (PLQYs), and solution processability, promote the perovskites to become a competitive candidate for the next-generation light-emitting diodes (LEDs) [1-6]. Inspiringly, great progresses have been achieved in green, red, and near-infrared perovskite LEDs (PeLEDs) where impressive external quantum efficiencies (EQEs) exceeding 20\% are reported [7-9], showing a great potential toward practical application. Although substantial efforts have been made in enhancing the blue PeLED performances, and efficiencies over $10 \%$ are achieved [10-14], the performances still lag far behind with the efficient green, red, and near-infrared PeLED analogs. The moderate blue PeLED performances undoubtedly retard the applications of PeLEDs in full-color displays and white-light illumination. It is highly desirable to develop highly performed and stable blue PeLEDs.

Recently, blue perovskite emitters, such as mixed-halide polycrystalline perovskites $[11,15-19]$, perovskite nanocrystals (NCs) [5, 10, 20-27], 2D perovskite nanoplatelets [28-32], and quasi-two-dimensional (quasi-2D) perovskites [33-41], have experienced extensive attention for efficient blue PeLEDs. Particularly, there is a booming development for the device efficiencies of quasi-2D perovskites taking the advantages of the energy funneling effect assisted fast exciton transfer and recombination, and the facilely solution-processed perovskite fabrication [42-44]. Typically, the component of quasi-2D perovskite consists of inorganic $\mathrm{Pb}-\mathrm{Br}$ octahedral layer and organic spacer cation, which is generally named as $\mathrm{B}_{2}\left(\mathrm{APbBr}_{3}\right)_{n-1} \mathrm{PbBr}_{4}$, where $\mathrm{B}$ is an organic spacer cation (e.g., phenylethylammonium: $\mathrm{PEA}^{+}$), $\mathrm{A}$ is a monovalent cation (e.g., $\mathrm{Cs}^{+}$, formamidinium: $\mathrm{FA}^{+}$), and $n$ represents the number of $\mathrm{Pb}-\mathrm{Br}$ octahedral layers $[45,46]$. As we know, inevitable defects are generated during the crystallization of quasi-2D perovskites due to the ionic character of the hybrid perovskites. The defects are generally believed to be related to the ionic defects (e.g., halide vacancies) [47]. The halide vacancies can serve as nonradiative recombination centers, causing severe trapmediated nonradiative losses and thus low emission efficiency. The vacancy defects can be effectively suppressed by molecular passivating agents which provide coordination or ionic bonding to neutralize the charged vacancies [48, 49]. For example, the molecules containing functional groups such as $-\mathrm{NH}_{2}, \mathrm{C}=\mathrm{O}$, or $\mathrm{P}=\mathrm{O}$ can effectively passivate the vacancy defects of perovskite films by saturating the undercoordinated $\mathrm{Pb}$ sites through sharing the electron pairs with the empty orbital of undercoordinated $\mathrm{Pb}$ cations, thus contributing to high PLQYs for the perovskite films [48-50]. Specially, the phosphine oxide passivator of trioctylphosphine oxide (TOPO) was reported to boost the longest photoluminescence (PL) lifetime of polycrystalline perovskite films to over 8 microseconds which are comparable with the carrier lifetimes of single-crystal perovskite [51]. Meanwhile, TOPO was also reported to successfully passivate the green $\mathrm{PEA}_{2}\left(\mathrm{FAPbBr}_{3}\right)_{\mathrm{n}-1} \mathrm{PbBr}_{4}$ quasi-2D perovskite surface defects for bright emission and the subsequent efficient green PeLEDs [52], showing the great potential of phosphine oxide passivators for the preparation of highperformance PeLEDs.

Quasi-2D perovskites usually contain a mixture of layered perovskites with phase distribution from the low- $n$ to high- $n$ phases $[45,46]$. This is because that there is a complex mixture of colloids with random size distribution in the precursor solution [53], which can act as nucleation centers to form $2 \mathrm{D}$ perovskites with different layers. In quasi-2D perovskites, the efficient energy transfer from the larger bandgap phase (i.e., lower- $n$ ) to the smaller bandgap phase (i.e., higher- $n$ ) is essential to realize the high emission efficiency $[37,52,54]$. Specially, different from the green-emissive quasi-2D perovskites, more organic spacer cations are needed to narrow the potential well of $\mathrm{Pb}-\mathrm{Br}$ octahedral layers for blue emission $[46,55]$. In other words, there are massive components of organic spacer cations in blue-emissive quasi-2D perovskites, which are harmful to the carrier transfer due to the insulating nature of the organic spacer cations [34, 55]. Meanwhile, there is a concern that the insulating carbon chain of phosphine oxide passivator will further aggravate the inefficient carrier transfer process; that is to say, the TOPO passivator may not be the ideal candidate for achieving efficient blue quasi-2D PeLEDs from its long carbon chain point of view. Consequently, it is rational and very important to optimize the passivating agents by promoting the carrier transfer in quasi-2D perovskites for high emission efficiency.

In this work, we systematically study the effects of phosphine oxide passivating agents of triethylphosphine oxide 
(TEPO), tributylphosphine oxide (TBPO), and trioctylphosphine oxide (TOPO) on the optoelectronic properties of quasi-2D perovskite films and demonstrate the TBPO-incorporated perovskites for the best-performed blue PeLEDs. Our results show that the appropriate carbon chain length of TBPO simultaneously offers good carrier confinement for the massive radiative recombination within the perovskites, and efficient carrier transfer in quasi-2D perovskites for high emission efficiency. Benefitting from the good optical and electrical properties of TBPO-incorporated perovskites, we achieve high-efficient blue PeLEDs with an EQE of $11.5 \%$ and prolonged operational stability of $41.1 \mathrm{~min}$, which is among the best-performing blue PeLEDs. This work contributes an effective approach to design effective passivation molecules for high-quality perovskite films and efficient blue PeLEDs.

\section{Experimental Section}

\subsection{Materials}

Propylamine hydrobromide (PABr, 99.999\%) and phenethylammonium hydrobromide (PEABr, 99.999\%) were purchased from Greatcell Solar; cesium bromide $(\mathrm{CsBr}$, 99.999\%) and lead bromide $\left(\mathrm{PbBr}_{2}, 99.999 \%\right)$ were bought from Sigma-Aldrich. Poly(9-vinylcarbazole) (PVK), tris(1phenyl-1H-benzimidazol-2-yl)benzene (TPBi, 99.5\%), and LiF (99.99\%) were purchased from Lumtec; triethylphosphine oxide (TEPO, 98\%), tributylphosphine oxide (TBPO, 98\%), trioctylphosphine oxide (TOPO, 99\%), and ethyl acetate (super dry, 99.9\%) were purchased from J\&K Chemical Ltd.

\subsection{Perovskite Emission Layer Preparation}

The precursor of quasi-2D $\mathrm{PEA}_{x} \mathrm{PA}_{2-x}\left(\mathrm{CsPbBr}_{3}\right)_{n-1} \mathrm{PbBr}_{4}$ perovskite was prepared by dissolving $\mathrm{CsBr}, \mathrm{PbBr}_{2}, \mathrm{PABr}$, and PEABr with a molar ratio of 1:1.1:1:0.2 into DMSO at a concentration of $0.15 \mathrm{~mol} \mathrm{~L}^{-1}$ (i.e., $0.15 \mathrm{M} \mathrm{CsBr}$ ). Then, the pristine perovskite films were fabricated by dynamically spin-coating the precursor at $3000 \mathrm{rpm}$ for $60 \mathrm{~s}$. Specifically, $100 \mu \mathrm{L}$ perovskite precursor was quickly dropped on the PVK substrate once the speed rate reaches $3000 \mathrm{rpm}$. Then, $300 \mu \mathrm{L}$ of ethyl acetate was quickly dripped on the substrate at $26 \mathrm{~s}$ after the spin-procedure starting, followed by annealing at $70{ }^{\circ} \mathrm{C}$ for $10 \mathrm{~min}$. For the phosphine oxidesincorporated perovskites, the preparation procedure is the same as the pristine perovskites, except for the antisolvent in which different concentrations of phosphine oxides were added.

\subsection{Device Fabrication}

The indium tin oxide (ITO) substrates were successively washed with deionized water, acetone, and isopropyl alcohol and then further treated with oxygen plasma for 5 min before use. The PVK layer was prepared by spin-coating the PVK solution ( $4 \mathrm{mg} \mathrm{mL}^{-1}$ in chlorobenzene) on ITO substrates at $4000 \mathrm{rpm}$ for $45 \mathrm{~s}$ and then heated at $120{ }^{\circ} \mathrm{C}$ for $15 \mathrm{~min}$, according to our previous work [44]. Then, the perovskite precursor solution was spin-coated on PVK substrates to prepare the perovskite films. Finally, TPBi, LiF, and $\mathrm{Al}$ electrodes were successively evaporated onto the perovskite films with the thickness of 45, 1, and $90 \mathrm{~nm}$ with deposition rates of $0.5,0.04$, and $1 \AA \mathrm{s}^{-1}$, respectively. After that, the devices were sealed by an ultraviolet-curable resin before testing.

\subsection{Characterization}

UV-Vis absorption spectra were carried out on the spectrophotometer of PERSEE TU-1901. The cross-sectional SEM characterization and the perovskite film morphologies were obtained using the scanning electron microscope (Zeiss 1550). The XPS measurements were performed with the use of Kratos Ultra Spectrometer equipped with monochromatized Al K $\alpha$ X-ray photons discharge lamp. The depthprofiling XPS signals of the perovskite films were performed by etching the perovskite films with argon ions. The PLQYs were obtained using the equipment of Hamamatsu Quantaurus-QY, model No. C11347. TA spectra were obtained with an ExciPro XL Femtosecond Transient Absorption Pump-Probe Spectrometer (CDP systems). The perovskite films were pumped with a femtosecond $365 \mathrm{~nm}$ laser pulse generated from an optical parametric amplifier. The probe pulses with a range from 380 to $800 \mathrm{~nm}$ were generated from the fundamental $800 \mathrm{~nm}$ laser pulses by focusing a small portion $(\approx 5 \mu \mathrm{J})$ into a 2 -mm-thick $\mathrm{CaF}_{2}$ plate. The PL and TRPL spectra of the perovskites were performed on PicoQaunt FluoTime 300 equipped with a picosecond 
pulse laser (360 nm, LDH-P-C-360). The capacitance-voltage $(C-V)$ measurements were recorded using a Keithley 4200A parameter analyzer at $1 \mathrm{kHz}$ with an AC amplitude of $20 \mathrm{mV}$. The capacitance-frequency $(C-f)$ measurements were performed using Keithley 4200A parameter analyzer with the frequency range from $10^{3}$ to $10^{5} \mathrm{~Hz}$ and an $\mathrm{AC}$ amplitude of $25 \mathrm{mV}$. The value of absolute dielectric permittivity is $8.85 \times 10^{-12} \mathrm{~F} \mathrm{~m}^{-1}$, the device area is $0.04 \mathrm{~cm}^{2}$, and the thickness of the perovskite film is $40 \mathrm{~nm}$. The geometrical capacitances for pristine, pristine/TEPO, pristine/TBPO, and pristine/TOPO are 6.61, 5.79, 5.23, and $4.52 \mathrm{nF}$, corresponding to the calculated $\varepsilon$ of $7.47,6.54,5.91$, and 5.11, respectively. The current density-voltage-luminance curves were performed on Ocean Optics system equipped with a Keithley 2400 source meter, an integrating sphere (FOIS1), and an QE Pro spectrometer. The integrating sphere was calibrated with a radiometric calibration light source (HL3plus) before use. The active area for the devices is $4 \mathrm{~mm}^{2}$. The scanning rate is $0.1 \mathrm{~V} \mathrm{~s}^{-1}$ with a dwell time of $0.5 \mathrm{~s}$. The CIE coordinates of the devices were measured on LED Color Calculator (OSRAM).

\section{Results and Discussion}

Quasi-2D perovskites with mixed spacer cations have been demonstrated to be capable of fine modulation of the perovskite phase for efficient blue PeLEDs [35, 56, 57]. Herein, the quasi-2D perovskite of $\mathrm{PEA}_{x} \mathrm{PA}_{2-x}\left(\mathrm{CsPbBr}_{3}\right)_{n-1} \mathrm{PbBr}_{4}$ (PEA: phenylethylammonium, PA: propylammonium) is employed as the pristine perovskite due to the suppressed low- $n$ perovskite phases for fast energy transfer and thus decent blue PeLEDs as shown in our previous works [56]. The pristine perovskite was prepared by spin-coating the perovskite precursor on PVK substrate followed by an antisolvent process with ethyl acetate (EA). For the passivating agents of alkyl phosphine oxides-treated perovskite films, EA with triethylphosphine oxide (TEPO), tributylphosphine oxide (TBPO), or trioctylphosphine oxide (TOPO) as an antisolvent was dropped on the perovskite films during the antisolvent process as illustrated in Fig. 1a. Details are shown in the experimental section.

\subsection{Interactions Between the Phosphine Oxides and the Perovskites}

In TEPO, TBPO, and TOPO molecules, the $\mathrm{P}=\mathrm{O}$ functional group provides the electron pairs to coordinate the unsaturated $\mathrm{Pb}^{2+}$ of perovskite films for halide vacancy passivation. The interaction between $\mathrm{P}=\mathrm{O}$ and $\mathrm{Pb}^{2+}$ can be vividly verified by the Fourier-transform infrared spectroscopy (FT-IR) performed on TEPO, TBPO, TOPO, and their incorporated $\mathrm{PbBr}_{2}$ powder (abbreviated as TEPO/ $\mathrm{PbBr}_{2}, \mathrm{TBPO} / \mathrm{PbBr}_{2}$, and $\mathrm{TOPO} / \mathrm{PbBr}_{2}$ ). As shown in Fig. 1b-d, the characteristic $\mathrm{P}=\mathrm{O}$ stretching vibration peaks are located at 1132,1153 , and $1147 \mathrm{~cm}^{-1}$ for TEPO, TBPO, and TOPO [50, 52, 58], which shift to the lower wavenumbers to be 1106,1118 , and $1105 \mathrm{~cm}^{-1}$ for TEPO/PbBr $2, \mathrm{TBPO} / \mathrm{PbBr}_{2}$, and TOPO/ $\mathrm{PbBr}_{2}$, respectively. The shift of $\mathrm{P}=\mathrm{O}$ stretching vibration to that lower frequency reveals a decreased electron cloud density between $\mathrm{P}$ and $\mathrm{O}$ atoms due to the complexation between $\mathrm{P}=\mathrm{O}$ and $\mathrm{PbBr}_{2}$ through the oxygen atom of $\mathrm{P}=\mathrm{O}$ with the empty $6 \mathrm{p}$ orbital of $\mathrm{Pb}^{2+}$. In addition, X-ray photoelectron spectroscopy (XPS) was performed to clarify the interaction of the phosphine oxides with the $\mathrm{Pb}-\mathrm{Br}$ framework of perovskite films. As presented in Fig. 1e, the peaks centered at 143.5 and $138.6 \mathrm{eV}$ for the pristine perovskite film are assigned to $\mathrm{Pb} 4 f$ signal, which shift toward the lower binding energy upon incorporation of TEPO (pristine/TBPO), TBPO (pristine/TBPO), and TOPO (pristine/ TOPO). The reduced binding energy indicates the lowered oxidation state of lead owing to the electron donation from the oxygen of the phosphine oxides. In addition, the similar shifts of $\mathrm{Pb} 4 f$ peaks to the lower binding energy are observed when the pristine/TEPO, pristine/TBPO, and pristine/TOPO perovskite films are etched to a depth of $30 \mathrm{~nm}$ by argon ion during XPS measurement as described in experimental section, suggesting the permeation of TEPO, TBPO, and TOPO into perovskite films with the antisolvent treatment (Supplementary Fig. S1). Besides, two extra peaks at 141.1 and $136.2 \mathrm{eV}$ are observed in pristine perovskites, which are attributed to the signals of metallic lead [59]. As reported previously, the presence of metallic lead is associated with halide vacancies in the perovskite lattice $[59,60]$, while no metallic lead signal is detected in pristine/TEPO, pristine/TBPO, and pristine/TOPO perovskites, indicating that the halide vacancies are effectively suppressed. Accordingly, it is found that the pristine/TEPO, pristine/TBPO, and pristine/TOPO perovskites show higher PL intensity than 
(a) precursor solution

O
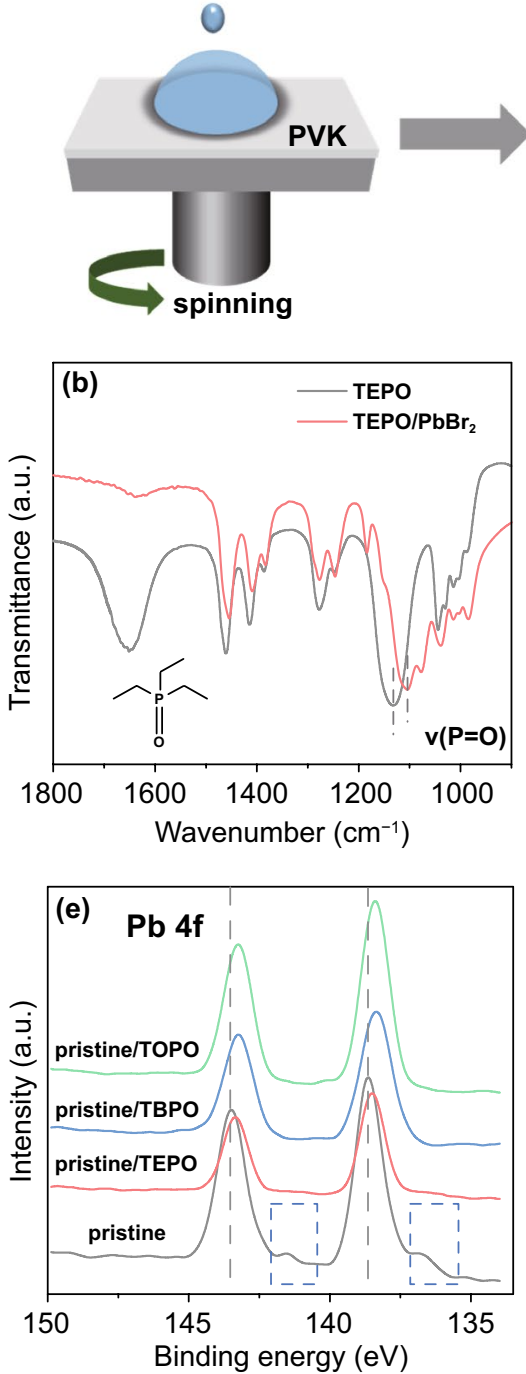
oxide

0
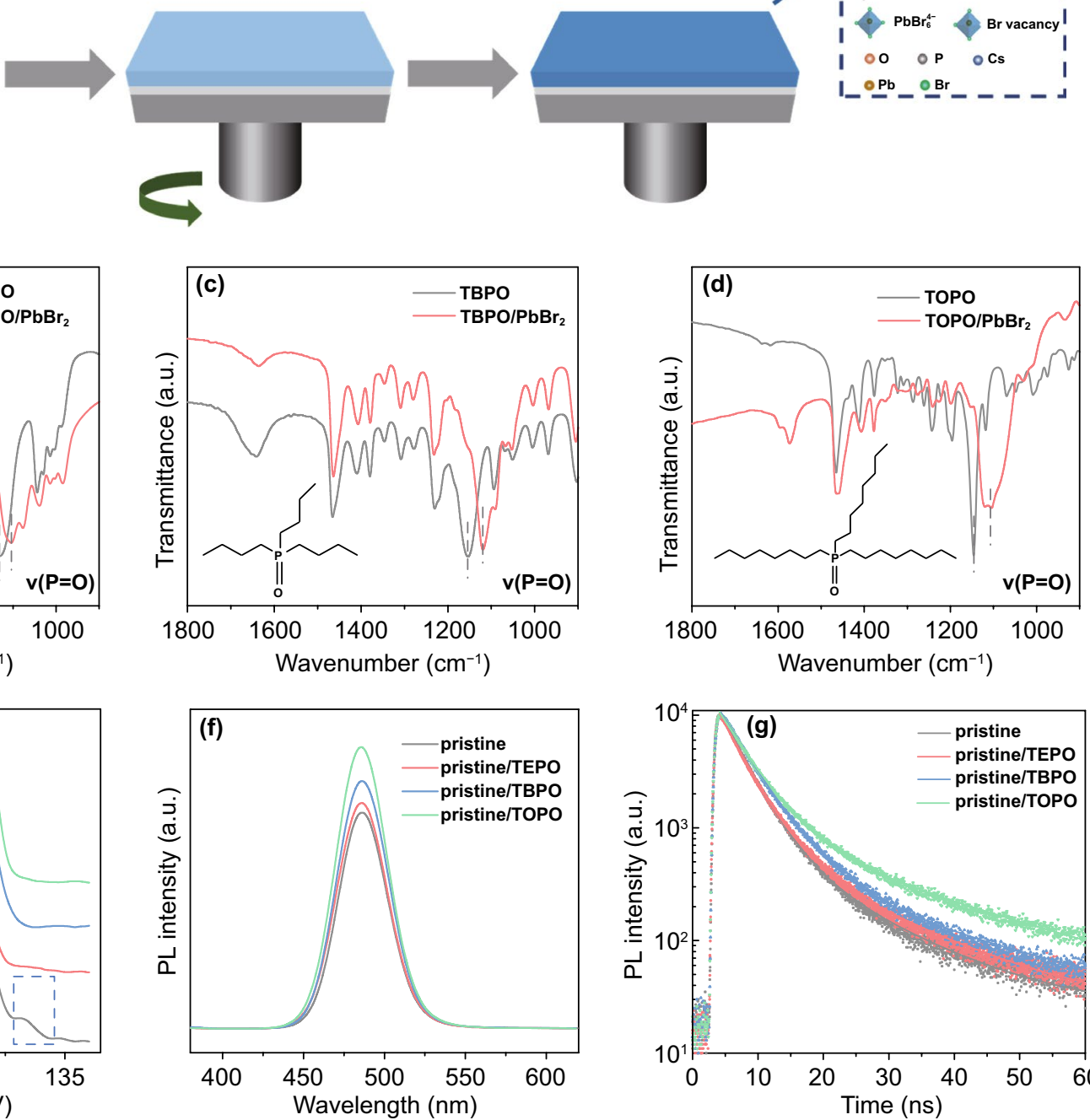

incorporade incorporated perovskite
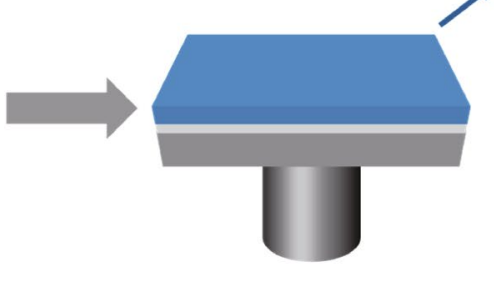

Tेo alkyl phosphine oxide

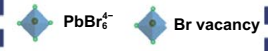

I OO OP OCs
$\left.\mathrm{I}_{-} \stackrel{\mathrm{Pb}}{-}-\stackrel{\mathrm{Br}}{-}---\right\lrcorner$

Fig. 1 a Schematic illustration of the preparation of the perovskite films. FT-IR spectroscopy and the chemical structure of $\mathbf{b}$ TEPO, $\mathbf{c}$ TBPO, and $\mathbf{d}$ TOPO and their incorporated $\mathrm{PbBr}_{2}$ powder. e XPS spectra of $\mathrm{Pb} 4 f$ signals, $\mathbf{f}$ steady-state photoluminescence, and $\mathbf{g}$ the time-resolved photoluminescence (TRPL) decay spectra of pristine and TEPO-, TBPO-, and TOPO-incorporated perovskite films

of pristine perovskite (Fig. 1f) with PLQYs increasing from $50.3 \%$ (pristine) to $56.4 \%$ (pristine/TEPO), $63.8 \%$ (pristine/ TBPO), and $69.8 \%$ (pristine/TOPO), respectively. These indicate the reduction of nonradiative recombination upon the incorporation of TEPO, TBPO, and TOPO. In addition, the perovskite PLQYs increase in the order of pristine/TEPO, pristine/TBPO, and pristine/TOPO perovskites, showing that the longer carbon chain length of the phosphine oxides is more effective in suppressing the nonradiative recombination loss, which will be discussed in the following part. Consequently, we show that there is a strong interaction between the phosphine oxides and the perovskites and thus effectively suppress the halide vacancies for less nonradiative recombination. 


\subsection{The Carrier Confinement Ability of the Phosphine Oxides}

To further study the effects of TEPO, TBPO, and TOPO on perovskite film carrier lifetime, the time-resolved PL (TRPL) measurements were performed on the phosphine oxides-incorporated perovskite films (Fig. 1g). The TRPL decay curves can be fitted by a biexponential function of $I(\mathrm{t})=A_{1} \exp \left(-t / \tau_{1}\right)+A_{2} \exp \left(-t / \tau_{2}\right)$, where $A_{1}, A_{2}$ are the amplitudes, and $\tau_{1}, \tau_{2}$ are time constants for fast and slow decay components, respectively. The fast decay component is related to trap-assisted nonradiative recombination at grain surfaces, and the slow decay component can be attributed to the radiative recombination within the perovskites [6, 61]. The extracted parameters from the TRPL decay curves are recorded in Supplementary Table S1. It is revealed that the perovskite PL lifetimes gradually increase with the incorporation of TEPO, TBPO, and TOPO. The fast decay lifetimes are raised from $3.53 \mathrm{~ns}$ (pristine) to 3.82 (pristine/ TEPO), 4.35 (pristine/TBPO), and $4.37 \mathrm{~ns}$ (pristine/TOPO), respectively, revealing the incorporation of phosphine oxides for reduced perovskite defects, consistent with the results of metallic lead suppression as shown in Fig. 1e. Meanwhile, the slow decay times greatly prolong from $12.81 \mathrm{~ns}$ (pristine) to 14.56 (pristine/TEPO), 19.82 (pristine/TBPO), and $22.08 \mathrm{~ns}$ (pristine/TOPO), respectively, which shows the strengthened radiative recombination within the phosphine oxides-incorporated perovskites. Notably, the slow decay times for pristine/TBPO and pristine/TOPO perovskites are much longer than pristine/TEPO perovskite, indicating the longer carbon chain of phosphine oxides for more radiative recombination within the perovskites. As illustrated in Fig. 2a, the phosphine oxides passivate the unsaturated $\mathrm{Pb}^{2+}$ through the coordination of the $\mathrm{P}=\mathrm{O}$ functional group while leaving the carbon chain at the perovskite surfaces and/or boundaries. The longer carbon chains of TBPO and

(a)
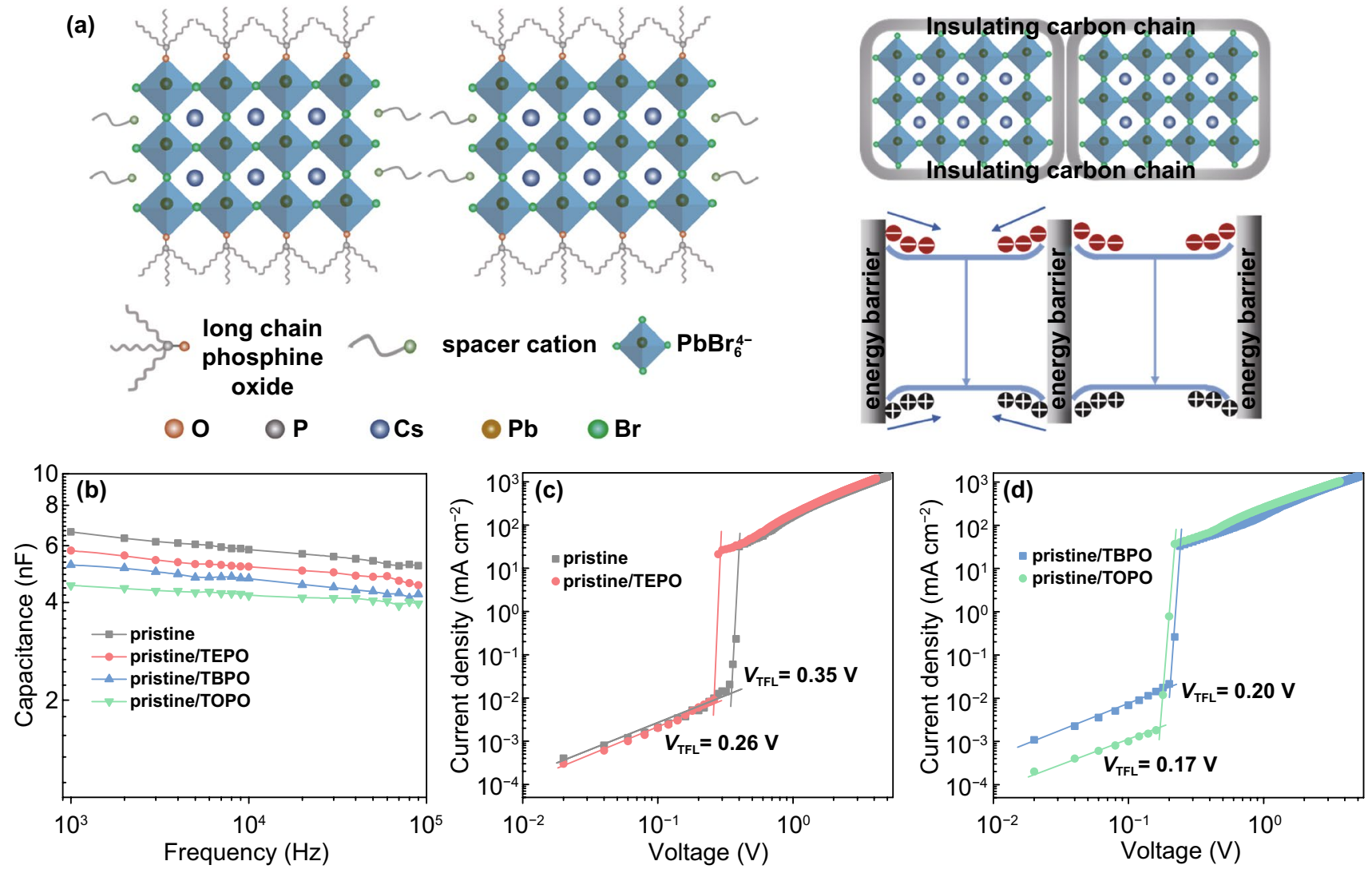

Fig. 2 a Schematic illustration of the phosphine oxides on perovskite surfaces and the function of carbon chain for carrier confinement. b Capacitance-frequency $(C-f)$ and $\mathbf{c}, \mathbf{d}$ space charge limited current (SCLC) measurements of pristine, TEPO-, TBPO-, and TOPO-incorporated perovskite devices 
TOPO than TEPO act as an energy barrier which confines the carriers inside the perovskite due to the insulating nature and low permittivity of the carbon chain and thus reduces the nonradiative recombination for bright emission. In addition, it is reported that the carrier confinement ability of the organic chains can be evaluated by the dielectric mismatch between the organic chains and the inorganic layer (e.g., $\mathrm{Pb}-\mathrm{Br}$ octahedral layer), and the smaller dielectric constant $(\varepsilon)$ of the phosphine oxides will cause a larger dielectric mismatch for better carrier confinement [62]. Specifically, when decreasing the dielectric constant of the phosphine oxides, there is a weakened dielectric screening effect around the perovskites, which results in strong Coulomb force between electron and hole of the exciton for more radiative recombination (Supplementary Fig. S2). Therefore, the smaller dielectric constant of the phosphine oxides will contribute to better carrier confinement. To study the $\varepsilon$ of the phosphine oxides, the capacitance-frequency $(C-f)$ measurements were performed on the devices with a structure of ITO/PVK/perovskite/Al (Supplementary Fig. S3). The $\varepsilon$ of the phosphine oxides can be compared from the $\varepsilon$ of their incorporated perovskites according to the equation of $\varepsilon=(C L) /\left(\varepsilon_{0} S\right)$, where $C$ is the geometrical capacitance, $L$ is the thickness of the perovskite film, $\varepsilon_{0}$ is the absolute dielectric permittivity, and $S$ is the device area. As shown in Fig. 2b, the perovskite capacitance decreases after incorporation of TEPO, TBPO, and TOPO, indicating the lowered $\varepsilon$ for the incorporated perovskite films. Furthermore, the calculated $\varepsilon$ for pristine/ TBPO and pristine/TOPO perovskites is 5.91 and 5.11, respectively, which are smaller than 7.47 and 6.54 of the pristine and pristine/TEPO perovskites. Since the dielectric constant of the perovskites changes depending on the incorporation of the phosphine oxides, the smaller $\varepsilon$ of pristine/ TBPO and pristine/TOPO perovskites implies the smaller $\varepsilon$ for TBPO and TOPO and thus contributes to better carrier confinement. Besides, to rule out the effect of the perovskite defect concentration on the evaluation of the organic dielectric constant, the capacitance-frequency measurements were performed on the samples without perovskite precursors. The TBPO- and TOPO-incorporated films show lower capacitance than that of TEPO-incorporated one at the same preparation condition, indicating their smaller dielectric constants (Supplementary Fig. S4). In addition, the small $\varepsilon$ values for pristine/TBPO and pristine/TOPO perovskites, together with the effective defect passivation roles of $\mathrm{P}=\mathrm{O}$ function group, promote a small trap state density for the perovskites, which can be obtained from the space charge limited current (SCLC) measurements of the devices (ITO/ perovskite/ $\left.\mathrm{MoO}_{3} / \mathrm{Ag}\right)$ by the equation of $N=\left(2 V_{\mathrm{TFL}} \varepsilon_{\mathrm{r}} \varepsilon_{0}\right) /$ $\left(e L^{2}\right)$, where $N$ represents the trap state density, $V_{\mathrm{TFL}}$ is the trap-filled limit voltage, and $\varepsilon_{\mathrm{r}}$ is the dielectric constant of the perovskites (Fig. 2c, d). The trap state density for pristine/TBPO and pristine/TOPO perovskites is $5.99 \times 10^{16}$ and $8.16 \times 10^{16} \mathrm{~cm}^{-3}$, respectively, which are much smaller than $1.81 \times 10^{17}$ and $1.17 \times 10^{17} \mathrm{~cm}^{-3}$ of the pristine and pristine/TEPO perovskites. Consequently, we show that the phosphine oxides can passivate the unsaturated $\mathrm{Pb}^{2+}$ of perovskite films through $\mathrm{P}=\mathrm{O}$ functional group. Meanwhile, we demonstrate that the longer carbon chain of TBPO and TOPO than TEPO promotes more radiative recombination internal the perovskites due to the better carrier confinement induced by the insulating nature and low permittivity of the longer carbon chain. However, it is noted that the insulating nature of the carbon chain may also cause some problems (e.g., inefficient energy transfer in the quasi-2D perovskite, poor carrier injection, and transport in the PeLEDs), which will hinder the improvement of the device efficiency. Further characterizations of the optoelectronic properties for pristine/TBPO and pristine/TOPO perovskites will be studied in the following part.

\subsection{Energy Transfers in the Phosphine Oxides Incorporated Quasi-2D Perovskites}

In quasi-2D perovskites, the photogenerated carriers are driven from a larger bandgap (i.e., low- $n$ ) phase to a smaller bandgap (i.e., high- $n$ ) phase and, subsequently, recombine in the high- $n$ phase (Fig. 3a) due to the energy funneling effect [46]. Therefore, an efficient energy transfer from low- $n$ phase to high- $n$ phase in the quasi-2D perovskite is vital for high emission efficiency. To study the photogenerated carrier transfer dynamics in the pristine/ TBPO and pristine/TOPO perovskites, the characterization of ultrafast transient absorption (TA) spectroscopy has been conducted. Figure 3b, c shows the TA spectra of pristine/TBPO and pristine/TOPO perovskites at different decay times, respectively. Three distinctive ground-state photobleaching (PB) signals can be observed at around 429,453 , and $480 \mathrm{~nm}$, which are corresponding to $n=2$, $n=3$, and $n \geq 4$ phases $[35,44]$. The signals are consistent with their steady-state absorption spectra (Fig. 3d), where 

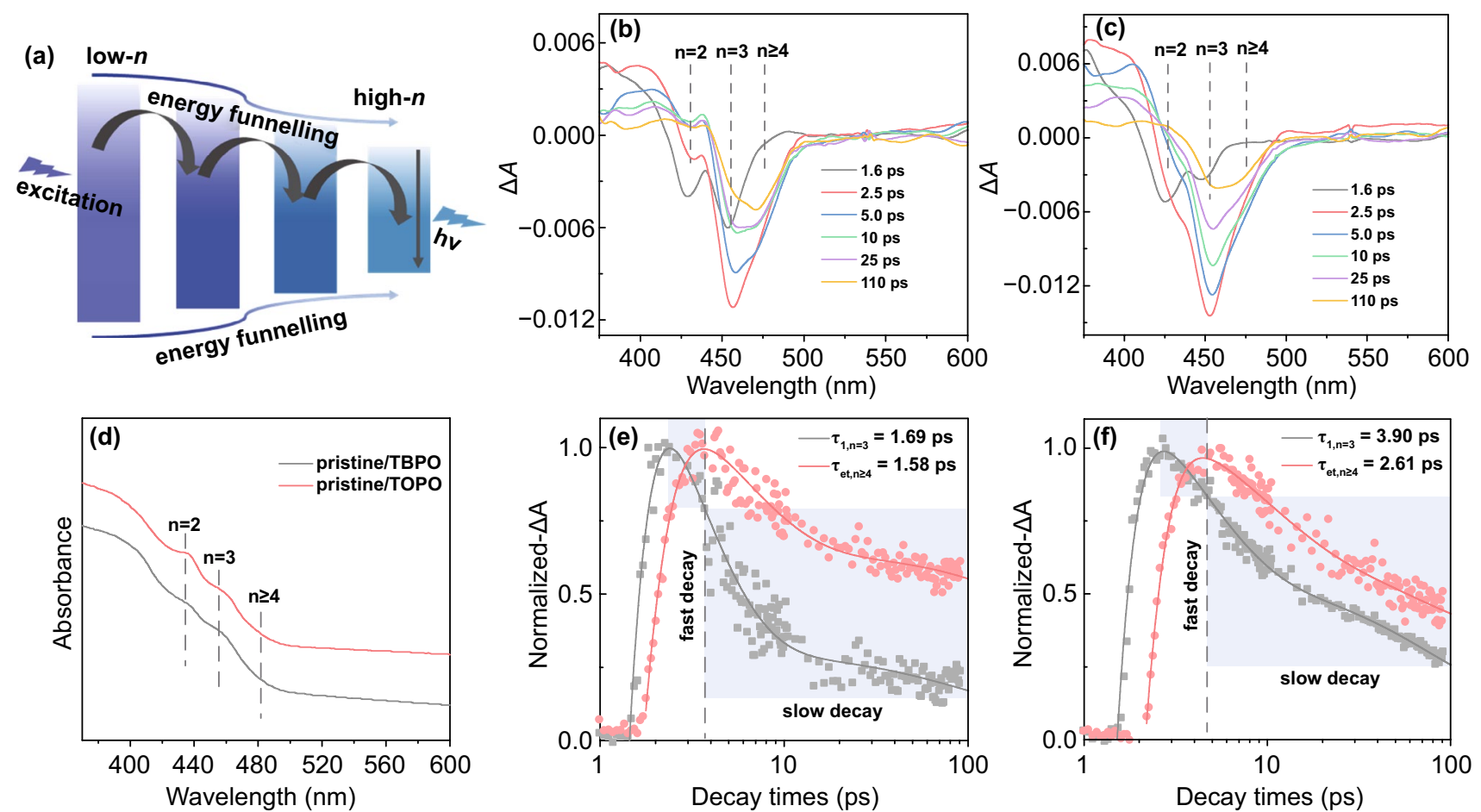

Fig. 3 a Schematic illustration of the energy transfer process in quasi-2D perovskites. Transient absorption (TA) spectra of $\mathbf{b}$ pristine/TBPO and c pristine/TOPO perovskite films at different delay times. d The UV-visible absorption spectra of pristine/TBPO and pristine/TOPO perovskites. The extracted kinetics of the TA signals at selected wavelengths (453 and $480 \mathrm{~nm}$ ) for e pristine/TBPO and $\mathbf{f}$ pristine/TOPO perovskite films

two shoulders of the absorption spectra centered at around 429 and $453 \mathrm{~nm}$ and a red-tail extending to $\approx 480 \mathrm{~nm}$ are observed. Besides, it is found that the photogenerated carriers are initially generated in low-order (i.e., $n=2$, $n=3$ ) phases with a fast buildup of $n=2$ and $n=3 \mathrm{~PB}$ peaks in pristine/TBPO and pristine/TOPO perovskites. With increase in the decay time, the signals of low-order phases decrease. Meanwhile, the high-order (i.e., $n \geq 4$ ) PB peak gradually increases which indicates the energy transfer from low-order phases to high-order ones [37]. To obtain more details of the carrier transfer process, we extract the time traces of low-order (e.g., $n=3,453 \mathrm{~nm}$ ) phase and high-order (e.g., $n \geq 4,480 \mathrm{~nm}$ ) phase from the decay kinetics (Fig. 3e, f). The kinetics of each PB can be fitted by a multiexponential function: $\Delta A(\mathrm{t})=a_{1}$ $\exp \left(-t / \tau_{1}\right)+a_{2} \exp \left(-t / \tau_{2}\right)+a_{3} \exp \left(-t / \tau_{3}\right)-c_{1} \exp \left(-t / \tau_{\mathrm{et}}\right)$, where $a_{1}, a_{2}, a_{3}$, and $c_{1}$ are amplitudes, $\tau_{1}$ represents the fast decay time constant due to the carrier transfer process between perovskite phases, $\tau_{2}$ and $\tau_{3}$ represent the slow decay time constant, and $\tau_{\text {et }}$ is the formation time constant obtained from the raising component of the PB signal [37, 63]. The fitting parameters are extracted in Supplementary
Table S2. The PB of $n=3$ phase shows a fast decay time with $\tau_{1}$ of $3.90 \mathrm{ps}$ for pristine/TOPO perovskite, which closely matches with the $\tau_{\text {et }}(2.61 \mathrm{ps})$ of the $n \geq 4$ phase PB (Fig. 3f), indicating a fast carrier transfer process from $n=3$ phase to $n \geq 4$ phase. However, the $\tau_{1}$ decreases to 1.69 ps for pristine/TBPO perovskite (Fig. 3e), which is less than half of pristine/TOPO perovskite ( $3.90 \mathrm{ps})$, indicating more efficient energy transfer from the low-order phase $(n=3)$ to the high-order phase $(n \geq 4)$. Meanwhile, the $\tau_{\text {et }}$ of $n \geq 4$ phase for pristine/TBPO perovskite is $1.58 \mathrm{ps}$ which is also smaller than that of pristine/TOPO perovskite $(2.61 \mathrm{ps})$, further verifying the faster energy transfer process in pristine/TBPO perovskite. In consequence, the TA measurements show that pristine/TBPO perovskites have more efficient energy transfer from the low-order phase $(n=3)$ to the high-order phase $(n \geq 4)$ than that of pristine/TOPO perovskite, which is beneficial to high device performance as will be discussed later. 


\subsection{Device Performances of the Phosphine Oxides Incorporated Blue PeLEDs}

The PeLEDs were fabricated to evaluate the electroluminescence (EL) performances of the phosphine oxidesincorporated perovskites. The devices are assembled with the structure of ITO/PVK/perovskite/tris(1-phenyl1H-benzimidazol-2-yl)benzene (TPBi)/LiF/Al, in which the perovskite emission layer is sandwiched between the hole injection layer (PVK) and the electron injection layer
(TPBi) as illustrated in Fig. 4a. The cross-sectional scanning electron microscopy (SEM) image of the device is displayed in Fig. 4b. The thicknesses of PVK, perovskite, TPBi, and $\mathrm{LiF} / \mathrm{Al}$ are around 20, 40, 45, and $90 \mathrm{~nm}$, respectively. Meanwhile, the SEM images show uniform perovskite films with free pinholes for pristine/TBPO and pristine/TOPO perovskites (Supplementary Fig. S5).

The maximum luminance of pristine PeLEDs in Fig. $4 \mathrm{~d}$ is $761 \mathrm{~cd} \mathrm{~m}^{-2}$ under the bias of $5.3 \mathrm{~V}$, which increases to 868 and $1119 \mathrm{~cd} \mathrm{~m}^{-2}$ for pristine/TOPO and pristine/TBPO
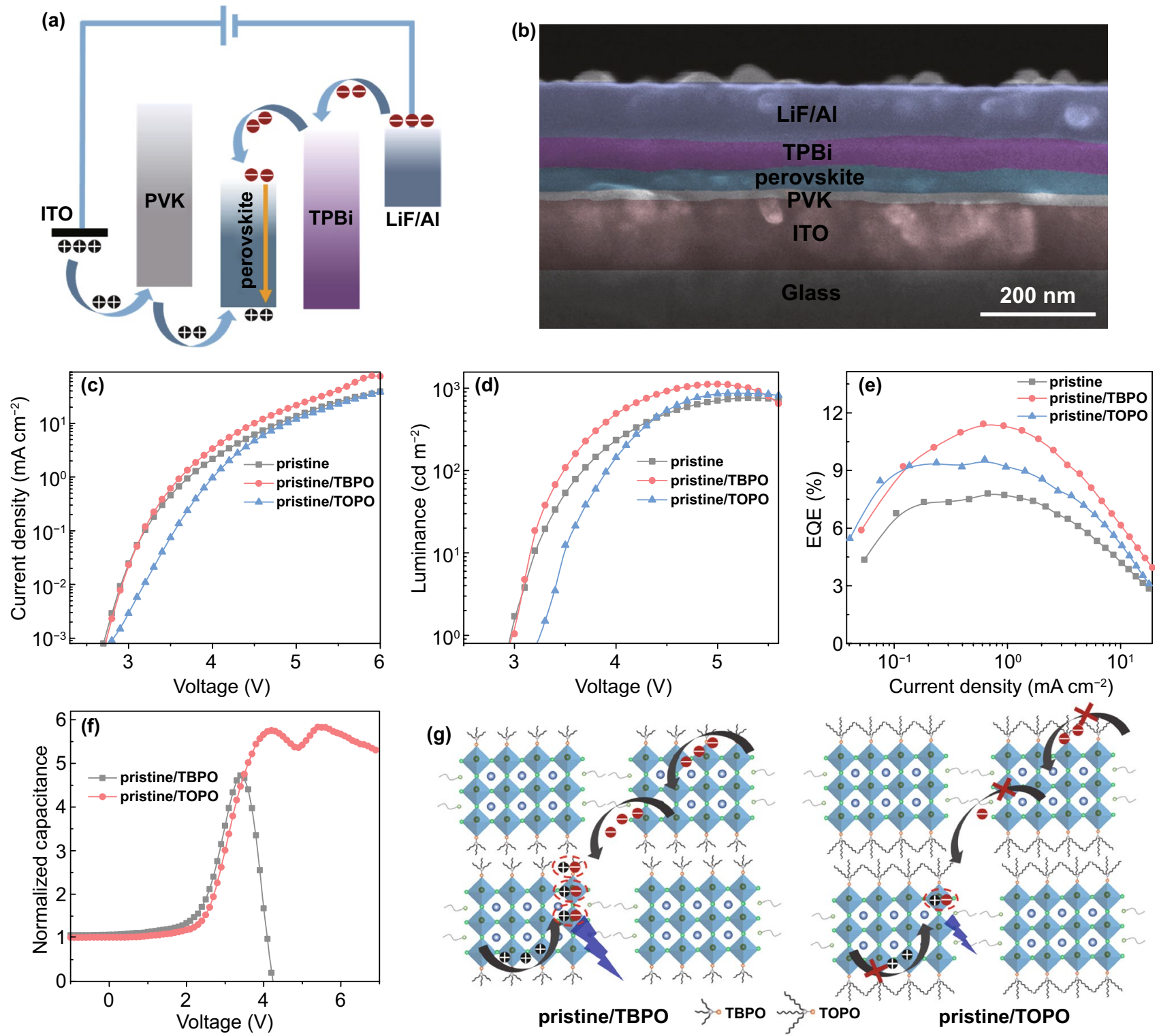

Fig. 4 a Schematic illustration of the PeLED structure, $\mathbf{b}$ the cross section scanning electron microscopy (SEM) image of PeLEDs, and PeLED performance: c current density-voltage $(J-V)$, d luminance-voltage $(L-V)$, and e efficiency-current density $(E Q E-J)$ curves. f The device capacitance-voltage $(C-V)$ measurements and $\mathbf{g}$ the illustration of charge transfer process in pristine/TBPO and pristine/TOPO PeLEDs 
devices. Accordingly, the EQE and current efficiency (CE) are dramatically enhanced from $7.8 \%$ and $11.8 \mathrm{~cd} \mathrm{~A}^{-1}$ (pristine) to $9.6 \%$ and $15.7 \mathrm{~cd} \mathrm{~A}^{-1}$ (pristine/TOPO), and $11.5 \%$ and $17.7 \mathrm{~cd} \mathrm{~A}^{-1}$ (pristine/TBPO), respectively (Fig. 4e; Supplementary S6). The improved pristine/TOPO and pristine/ TBPO device performances are further verified by the decent average EQEs which increase from 6.9\% (pristine) to 8.3\% (pristine/TOPO) and $10.4 \%$ (pristine/TBPO), respectively, extracted from 15 devices for each condition (Supplementary Fig. S7). The improvements can be attributed to the effective defect passivation and carrier confinement of TBPO and TOPO for massive radiative recombination. Importantly, the pristine/TBPO PeLEDs show higher efficiency than pristine/ TOPO devices, benefitting from the more efficient energy transfer from the low-order phase to the high-order phase in pristine/TBPO perovskite, as shown in TA spectra (Fig. 3e). Furthermore, it is found that the pristine/TOPO PeLEDs exhibit smaller current density (Fig. 4c) and larger turn-on voltage (corresponding to a luminance of $1 \mathrm{~cd} \mathrm{~m}^{-2}$ ) (Fig. 4d) than that of pristine/TBPO PeLEDs, which reveal a larger carrier injection barrier for pristine/TOPO PeLEDs and thus show only moderate improvement of the device efficiency. To further verify the charge injection and recombination capacity of pristine/TBPO and pristine/TOPO PeLEDs, the capacitance-voltage $(C-V)$ measurements of the devices were performed (Fig. 4f). It is observed that the capacitances of pristine/TBPO and pristine/TOPO devices show different trends against the bias voltages. In the $C-V$ curves, the capacitance increases with the raise of the bias voltage, indicating that the charges are injected into the devices, while the capacitance of pristine/TBPO devices rises faster than that of pristine/TOPO devices, indicating more charges are injected. When further increasing the voltages, the capacitance of pristine/TBPO devices reaches the peak value and then sharply declines due to the largely reduced charges caused by the radiative recombination of massive electrons and holes [64]. Differently, the capacitance of pristine/TOPO devices drops slightly after the peak capacitance then rises again. This variation vividly illustrates the less efficient radiative recombination of holes and electrons in pristine/ TOPO PeLEDs. The results well agree with the moderate brightness of $868 \mathrm{~cd} \mathrm{~m}^{-2}$ for pristine/TOPO devices, which is smaller than that of pristine/TBPO devices $\left(1119 \mathrm{~cd} \mathrm{~m}^{-2}\right)$. The decent role of TBPO for improved brightness can be further applied to the green PeLEDs (Supplementary Fig. S8), in which the pristine/TBPO PeLEDs show much higher brightness of $3411 \mathrm{~cd} \mathrm{~m}^{-2}$ than that of pristine PeLEDs $\left(2135 \mathrm{~cd} \mathrm{~m}^{-2}\right.$ ), indicating the general role of TBPO for high device performance. We also investigate the effect of different TBPO and TOPO concentrations on the PeLED performances (Supplementary Figs. S9 and S10), in which the highest efficiencies are obtained for $2 \mathrm{mM}$ TBPO- and TOPO-incorporated PeLEDs. The performance will decline at a higher concentration. Particularly, the TOPO-incorporated PeLEDs show a large decrease in the efficiency with the increase in the concentration to $3 \mathrm{mM}$, which can be attributed to the enlarged injection barrier as evidenced by the increased turn-on voltage (Supplementary Fig. S10b). Besides, the pristine/TEPO PeLEDs are also fabricated and the efficiency distribution extracted from 15 devices is shown in Supplementary Fig. S11. The highest efficiency of pristine/TEPO PeLEDs is $8.9 \%$ with an average of $7.9 \%$ which is lower than that of pristine/TBPO (10.4\%) and pristine/TOPO (8.3\%) devices due to the weak carrier confinement as shown previously.

The EL spectra of pristine, pristine/TOPO, and pristine/ TBPO devices are centered at around $488 \mathrm{~nm}$ (Fig. 5a), which are consistent with their PL spectra, indicating that the EL is merely derived from the perovskites. Besides, the EL peak wavelength remains unchanged with the increase in bias voltage from 3.5 to $6.0 \mathrm{~V}$ (Fig. 5b; Supplementary S12) and thus achieves good spectral stability for the PeLEDs as verified by the neglectable variation of the corresponding Commission Internationale de L'Eclairage (CIE) coordinates (Supplementary Fig. S13, Table S3). Meanwhile, the pristine/TBPO PeLEDs show a narrowband emission with the full width at half maximum (FWHM) of around $25 \mathrm{~nm}$, which enables a good color purity with CIE chromaticity coordinate at $(0.078,0.28)$ (Fig. $5 \mathrm{c})$. Regarding operational stability, the lifetime of the PeLEDs was measured under a continuous constant current $\left(0.4 \mathrm{~mA} \mathrm{~cm}^{-2}\right)$ since LEDs are usually current driven devices. The lifetime $\left(T_{50}\right)$ defines as the working time of PeLED decaying to $50 \%$ of its initial luminance $\left(L_{0}\right)$. The lifetime for pristine/TBPO PeLEDs reaches $41.1 \mathrm{~min}$ at the initial luminance of $33 \mathrm{~cd} \mathrm{~m}^{-2}$ (Fig. 5d), which is longer than that of pristine (15.9 min, $L_{0}$ : $21 \mathrm{~cd} \mathrm{~m}^{-2}$ ) and pristine/TOPO (27.3 $\mathrm{min}, L_{0}: 28 \mathrm{~cd} \mathrm{~m}^{-2}$ ) and represents one of the most stable blue PeLEDs [23, 25, 35, 37, 57, 65-71]. Meanwhile, the EL spectra of pristine/ TBPO PeLEDs keep unchanged and exhibit excellent stability under continuous operation (Fig. 5e). It is observed that the bias voltage of pristine/TBPO PeLEDs increases 

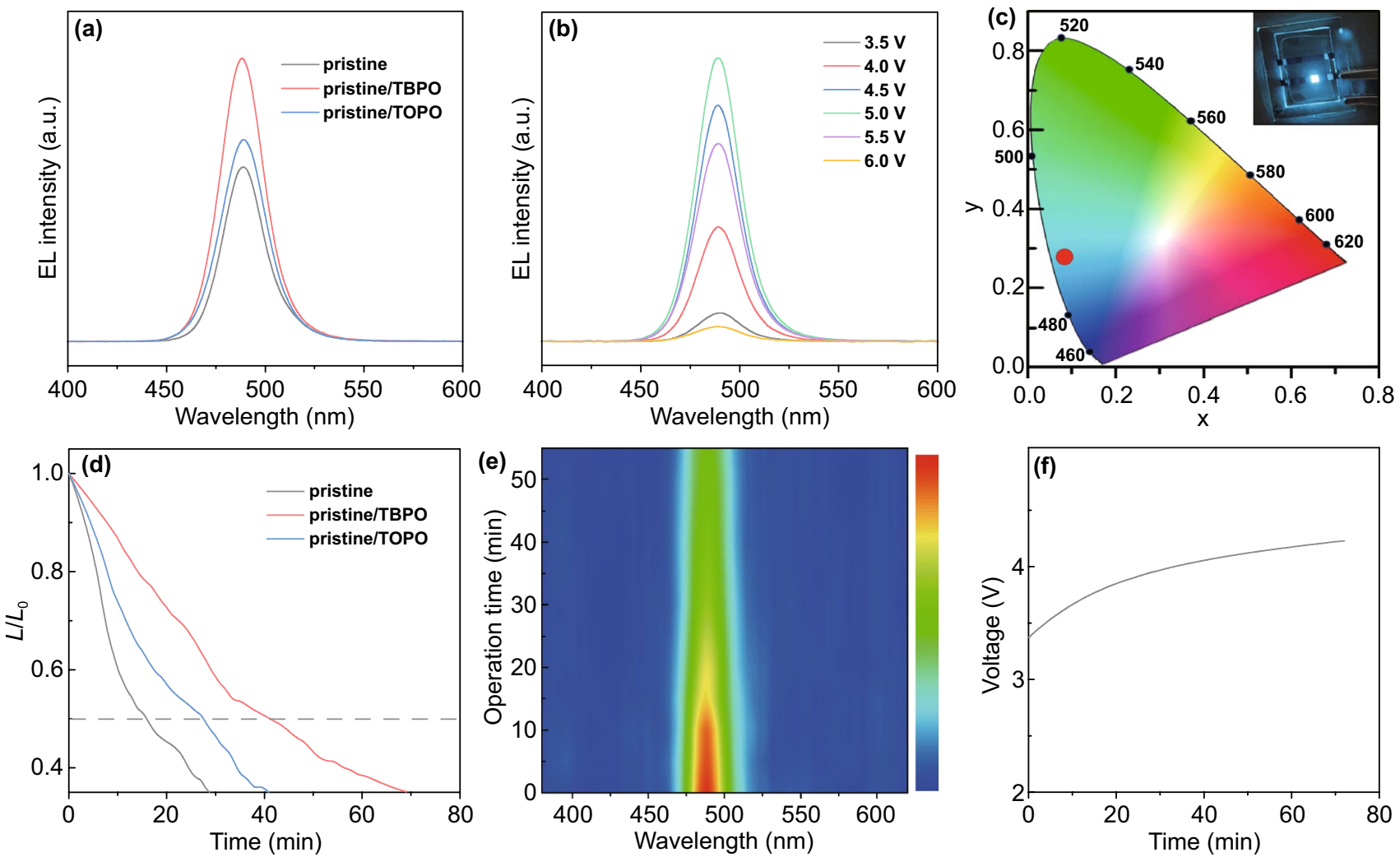

Fig. 5 a EL spectra of pristine, pristine/TBPO, and pristine/TBPO PeLEDs. b EL spectra of pristine/TBPO PeLEDs at different bias voltages and $\mathbf{c}$ the CIE coordinate of pristine/TBPO PeLEDs with the emission image in the inset. $\mathbf{d}$ The time-dependent stability lifetime measurements under $0.4 \mathrm{~mA} \mathrm{~cm}^{-2}$ of pristine, pristine/TBPO, and pristine/TBPO PeLEDs. e The traced EL spectra of pristine/TBPO PeLEDs during operation and $\mathbf{f}$ the corresponding bias voltage traces during the lifetime test

with prolonging the operation time (Fig. 5f) due to the increased device resistance during operation. We also measured the device lifetime under a larger injection current of $1.2 \mathrm{~mA} \mathrm{~cm}^{-2}$ as shown in Supplementary Fig. S14. The lifetime for pristine/TBPO is $11.2 \mathrm{~min}$ at the initial luminance of $102 \mathrm{~cd} \mathrm{~m}^{-2}$, much longer than that of 5.4 and $8.1 \mathrm{~min}$ for pristine and pristine/TOPO PeLEDs. Consequently, our results show that the pristine/TBPO PeLEDs have excellent spectral stability and the longest lifetime, which promotes a great potential for the blue PeLEDs toward practical applications.

\section{Conclusion}

In summary, we report to rationally study and optimize the passivating agents by controlling the carbon chain length to simultaneously enhance the carrier confinement for massive radiative recombination internal the perovskites and provide the efficient carrier transfer in quasi-2D perovskites. By further studying of the optoelectronic properties of the phosphine oxides (TEPO, TBPO, and TOPO)-incorporated perovskites, we demonstrate a good balance between carrier confinement and transfer for TBPO with the moderate chain length. TBPO offers better carrier confinement than TEPO and provides more efficient carrier transfer than TOPO. Based on the TBPO-incorporated perovskites, high-efficient blue PeLEDs with EQE up to $11.5 \%$ and operation lifetime as long as $41.1 \mathrm{~min}$ are achieved, which are among the bestperforming blue PeLEDs reported so far. Consequently, our work provides an effective way for the realization of highperformance and stable blue PeLEDs.

Acknowledgements This research was supported by National Key Research and Development Program (No. 2017YFE0120400), Equipment fund, Platform Research Fund and feed fund (Grant Nos. 2019157209 and 202011159254), from the University Grant Council of the University of Hong Kong, the General Research Fund (Grant Nos. 17200518, 17201819, and 17211220) and 
Collaboration Research Fund (C7035-20G) from Hong Kong Special Administrative Region, China, as well as the National Natural Science Foundation of China (Nos. 61875082 and 62105231) and the Natural Science Foundation of Jiangsu Province (No. BK20210712)

Funding Open access funding provided by Shanghai Jiao Tong University.

Open Access This article is licensed under a Creative Commons Attribution 4.0 International License, which permits use, sharing, adaptation, distribution and reproduction in any medium or format, as long as you give appropriate credit to the original author(s) and the source, provide a link to the Creative Commons licence, and indicate if changes were made. The images or other third party material in this article are included in the article's Creative Commons licence, unless indicated otherwise in a credit line to the material. If material is not included in the article's Creative Commons licence and your intended use is not permitted by statutory regulation or exceeds the permitted use, you will need to obtain permission directly from the copyright holder. To view a copy of this licence, visit http://creativecommons.org/licenses/by/4.0/.

Supplementary Information The online version contains supplementary material available at https://doi.org/10.1007/ s40820-022-00807-7.

\section{References}

1. L.N. Quan, B.P. Rand, R.H. Friend, S.G. Mhaisalkar, T.W. Lee et al., Perovskites for next-generation optical sources. Chem. Rev. 119(12), 7444-7477 (2019). https://doi.org/10.1021/acs. chemrev.9b00107

2. Z.K. Tan, R.S. Moghaddam, M.L. Lai, P. Docampo, R. Higler et al., Bright light-emitting diodes based on organometal halide perovskite. Nat. Nanotechnol. 9, 687-692 (2014). https:// doi.org/10.1038/nnano.2014.149

3. L. Protesescu, S. Yakunin, M.I. Bodnarchuk, F. Krieg, R. Caputo et al., Nanocrystals of cesium lead halide perovskites $\left(\mathrm{CsPbX}_{3}, \mathrm{X}=\mathrm{Cl}, \mathrm{Br}\right.$, and $\left.\mathrm{I}\right)$ : novel optoelectronic materials showing bright emission with wide color gamut. Nano Lett. 15(6), 3692-3696 (2015). https://doi.org/10.1021/n15048779

4. J. Wang, N. Wang, Y. Jin, J. Si, Z.K. Tan et al., Interfacial control toward efficient and low-voltage perovskite light-emitting diodes. Adv. Mater. 27(14), 2311-2316 (2015). https://doi.org/ 10.1002/adma.201405217

5. J. Song, J. Li, X. Li, L. Xu, Y. Dong et al., Quantum dot lightemitting diodes based on inorganic perovskite cesium lead halides $\left(\mathrm{CsPbX}_{3}\right)$. Adv. Mater. 27(44), 7162-7167 (2015). https://doi.org/10.1002/adma.201502567

6. H. Cho, S.H. Jeong, M.H. Park, Y.H. Kim, C. Wolf et al., Overcoming the electroluminescence efficiency limitations of perovskite light-emitting diodes. Science 350(6265), 1222 1225 (2015). https://doi.org/10.1126/science.aad1818

7. K. Lin, J. Xing, L.N. Quan, F.P.G. Arquer, X. Gong et al., Perovskite light-emitting diodes with external quantum efficiency exceeding 20 percent. Nature 562, 245-248 (2018). https://doi. org/10.1038/s41586-018-0575-3

8. Y. Cao, N. Wang, H. Tian, J. Guo, Y. Wei et al., Perovskite light-emitting diodes based on spontaneously formed submicrometre-scale structures. Nature 562, 249-253 (2018). https://doi.org/10.1038/s41586-018-0576-2

9. B. Zhao, S. Bai, V. Kim, R. Lamboll, R. Shivanna et al., Highefficiency perovskite-polymer bulk heterostructure light-emitting diodes. Nat. Photon. 12, 783-789 (2018). https://doi.org/ 10.1038/s41566-018-0283-4

10. Y. Dong, Y.K. Wang, F. Yuan, A. Johnston, Y. Liu et al., Bipolar-shell resurfacing for blue LEDs based on strongly confined perovskite quantum dots. Nat. Nanotechnol. 15, 668-674 (2020). https://doi.org/10.1038/s41565-020-0714-5

11. M. Karlsson, Z. Yi, S. Reichert, X. Luo, W. Lin et al., Mixed halide perovskites for spectrally stable and high-efficiency blue light-emitting diodes. Nat. Commun. 12, 361 (2021). https://doi.org/10.1038/s41467-020-20582-6

12. Q. Wang, X. Wang, Z. Yang, N. Zhou, Y. Deng et al., Efficient sky-blue perovskite light-emitting diodes via photoluminescence enhancement. Nat. Commun. 10, 5633 (2019). https://doi.org/10.1038/s41467-019-13580-w

13. Z. Chu, Y. Zhao, F. Ma, C.X. Zhang, H. Deng et al., Large cation ethylammonium incorporated perovskite for efficient and spectra stable blue light-emitting diodes. Nat. Commun. 11, 4165 (2020). https://doi.org/10.1038/ s41467-020-17943-6

14. Y. Liu, Z. Yu, S. Chen, J.H. Park, E.D. Jung et al., Boosting the efficiency of quasi-2D perovskites light-emitting diodes by using encapsulation growth method. Nano Energy 80, 105511 (2021). https://doi.org/10.1016/j.nanoen.2020.105511

15. N.K. Kumawat, A. Dey, A. Kumar, S.P. Gopinathan, K.L. Narasimhan et al., Band gap tuning of $\mathrm{CH}_{3} \mathrm{NH}_{3} \mathrm{~Pb}\left(\mathrm{Br}_{1-x} \mathrm{Cl}_{x}\right)_{3}$ hybrid perovskite for blue electroluminescence. ACS Appl. Mater. Interfaces 7(24), 13119-13124 (2015). https://doi.org/ 10.1021/acsami.5b02159

16. H.P. Kim, J. Kim, B.S. Kim, H.M. Kim, J. Kim et al., Highefficiency, blue, green, and near-infrared light-emitting diodes based on triple cation perovskite. Adv. Opt. Mater. 5(7), 1600920 (2017). https://doi.org/10.1002/adom.201600920

17. P. Du, J. Li, L. Wang, J. Liu, S. Li et al., Vacuum-deposited blue inorganic perovskite light-emitting diodes. ACS Appl. Mater. Interfaces 11(50), 47083-47090 (2019). https://doi.org/ 10.1021/acsami.9b17164

18. L. Cheng, C. Yi, Y. Tong, L. Zhu, G. Kusch et al., Halide homogenization for high-performance blue perovskite electroluminescence. Research 2020, 9017871 (2020). https://doi. org/10.34133/2020/9017871

19. H. Wang, Y. Xu, J. Wu, L. Chen, Q. Yang et al., Bright and color-stable blue-light-emitting diodes based on three-dimensional perovskite polycrystalline films via morphology and 
interface engineering. J. Phys. Chem. Lett. 11(4), 1411-1418 (2020). https://doi.org/10.1021/acs.jpclett.9b03714

20. J. Pan, L.N. Quan, Y. Zhao, W. Peng, B. Murali et al., Highly efficient perovskite-quantum-dot light-emitting diodes by surface engineering. Adv. Mater. 28(39), 8718-8725 (2016). https://doi.org/10.1002/adma.201600784

21. E.P. Yao, Z. Yang, L. Meng, P. Sun, S. Dong et al., Highbrightness blue and white LEDs based on inorganic perovskite nanocrystals and their composites. Adv. Mater. 29(23), 1606859 (2017). https://doi.org/10.1002/adma.201606859

22. S. Hou, M.K. Gangishetty, Q. Quan, D.N. Congreve, Efficient blue and white perovskite light-emitting diodes via manganese doping. Joule 2(11), 2421-2433 (2018). https://doi.org/ 10.1016/j.joule.2018.08.005

23. B.B. Zhang, S. Yuan, J.P. Ma, W. Zheng, H. Shen et al., General mild reaction creates highly luminescent organic-ligandlacking halide perovskite nanocrystals for efficient light-emitting diodes. J. Am. Chem. Soc. 141(38), 15423-15432 (2019). https://doi.org/10.1021/jacs.9b08140

24. F. Zhang, J. Song, B. Cai, X. Chen, C. Wei et al., Stabilizing electroluminescence color of blue perovskite LEDs via amine group doping. Sci. Bull. 66(21), 2189-2198 (2021). https://doi.org/10.1016/j.scib.2021.04.033

25 X. Zheng, S. Yuan, J. Liu, J. Yin, F. Yuan et al., Chlorine vacancy passivation in mixed halide perovskite quantum dots by organic pseudohalides enables efficient rec 2020 blue light-emitting diodes. ACS Energy Lett. 5(3), 793-798 (2020). https://doi.org/10.1021/acsenergylett.0c00057

26. W. Xu, R. Ji, P. Liu, L. Cheng, L. Zhu et al., In situ-fabricated perovskite nanocrystals for deep-blue light-emitting diodes. J. Phys. Chem. Lett. 11(24), 10348-10353 (2020). https://doi.org/10.1021/acs.jpclett.0c03120

27. C. Bi, Z. Yao, X. Sun, X. Wei, J. Wang et al., Perovskite quantum dots with ultralow trap density by acid etchingdriven ligand exchange for high luminance and stable pureblue light-emitting diodes. Adv. Mater. 33(15), 2006722 (2021). https://doi.org/10.1002/adma.202006722

28. D. Liang, Y. Peng, Y. Fu, M.J. Shearer, J. Zhang et al., Color-pure violet-light-emitting diodes based on layered lead halide perovskite nanoplates. ACS Nano 10(7), 68976904 (2016). https://doi.org/10.1021/acsnano.6b02683

29. Y. Wu, C. Wei, X. Li, Y. Li, S. Qiu et al., In situ passivation of $\mathrm{PbBr}_{6}{ }^{4}$ - octahedra toward blue luminescent $\mathrm{CsPbBr} 3$ nanoplatelets with near $100 \%$ absolute quantum yield. ACS Energy Lett. 3(9), 2030-2037 (2018). https://doi.org/10. 1021/acsenergylett.8b01025

30. C. Zhang, Q. Wan, B. Wang, W. Zheng, M. Liu et al., Surface ligand engineering toward brightly luminescent and stable cesium lead halide perovskite nanoplatelets for efficient blue-light-emitting diodes. J. Phys. Chem. C 123(43), 26161-26169 (2019). https://doi.org/10.1021/acs.jpcc. $9 \mathrm{~b} 09034$

31. S. Peng, Z. Wen, T. Ye, X. Xiao, K. Wang et al., Effective surface ligand-concentration tuning of deep-blue luminescent $\mathrm{FAPbBr} 3$ nanoplatelets with enhanced stability and charge transport. ACS Appl. Mater. Interfaces 12(28), 31863-31874 (2020). https://doi.org/10.1021/acsami.0c08552

32. W. Yin, M. Li, W. Dong, Z. Luo, Y. Li et al., Multidentate ligand polyethylenimine enables bright color-saturated blue light-emitting diodes based on $\mathrm{CsPbBr} 3$ nanoplatelets. ACS Energy Lett. 6(2), 477-484 (2021). https://doi.org/10.1021/ acsenergylett.0c02651

33. L. Cheng, Y. Cao, R. Ge, Y.Q. Wei, N.N. Wang et al., Sky-blue perovskite light-emitting diodes based on quasi-two-dimensional layered perovskites. Chin. Chem. Lett. 28(1), 29-31 (2017). https://doi.org/10.1016/j.cclet.2016.07.001

34. Q. Wang, J. Ren, X.F. Peng, X.X. Ji, X.H. Yang, Efficient sky-blue perovskite light-emitting devices based on ethylammonium bromide induced layered perovskites. ACS Appl. Mater. Interfaces 9(35), 29901-29906 (2017). https://doi.org/ 10.1021/acsami.7b07458

35. J. Xing, Y. Zhao, M. Askerka, L.N. Quan, X. Gong et al., Color-stable highly luminescent sky-blue perovskite lightemitting diodes. Nat. Commun. 9, 3541 (2018). https://doi. org/10.1038/s41467-018-05909-8

36. Z. Ren, X. Xiao, R. Ma, H. Lin, K. Wang et al., Hole transport bilayer structure for quasi-2D perovskite based blue light-emitting diodes with high brightness and good spectral stability. Adv. Funct. Mater. 29(43), 1905339 (2019). https:// doi.org/10.1002/adfm.201905339

37. Y. Jiang, C. Qin, M. Cui, T. He, K. Liu et al., Spectra stable blue perovskite light-emitting diodes. Nat. Commun. 10, 1868 (2019). https://doi.org/10.1038/s41467-019-09794-7

38. Z. Li, Z. Chen, Y. Yang, Q. Xue, H.L. Yip et al., Modulation of recombination zone position for quasi-two-dimensional blue perovskite light-emitting diodes with efficiency exceeding 5\%. Nat. Commun. 10, 1027 (2019). https://doi.org/10.1038/ s41467-019-09011-5

39. Y. Liu, J. Cui, K. Du, H. Tian, Z. He et al., Efficient blue light-emitting diodes based on quantum-confined bromide perovskite nanostructures. Nat. Photonics 13, 760-764 (2019). https://doi.org/10.1038/s41566-019-0505-4

40. C. Wang, D. Han, J. Wang, Y. Yang, X. Liu et al., Dimension control of in situ fabricated $\mathrm{CsPbClBr}_{2}$ nanocrystal films toward efficient blue light-emitting diodes. Nat. Commun. 11, 6428 (2020). https://doi.org/10.1038/s41467-020-20163-7

41. Y.K. Wang, D. Ma, F. Yuan, K. Singh, J.M. Pina et al., Chelating-agent-assisted control of $\mathrm{CsPbBr}_{3}$ quantum well growth enables stable blue perovskite emitters. Nat. Commun. 11, 3674 (2020). https://doi.org/10.1038/s41467-020-17482-0

42. M. Yuan, L.N. Quan, R. Comin, G. Walters, R. Sabatini et al., Perovskite energy funnels for efficient light-emitting diodes. Nat. Nanotechnol. 11, 872-877 (2016). https://doi.org/10. 1038/nnano.2016.110

43. P. Pang, G. Jin, C. Liang, B. Wang, W. Xiang et al., Rearranging low-dimensional phase distribution of quasi-2D perovskites for efficient sky-blue perovskite light-emitting diodes. ACS Nano 14(9), 11420-11430 (2020). https://doi.org/10. 1021/acsnano.0c03765

44. Z. Ren, J. Yu, Z. Qin, J. Wang, J. Sun et al., High-performance blue perovskite light-emitting diodes enabled by efficient 
energy transfer between coupled quasi-2D perovskite layers. Adv. Mater. 33(1), 202005570 (2021). https://doi.org/10.1002/ adma.202005570

45. L. Cheng, T. Jiang, Y. Cao, C. Yi, N. Wang et al., Multiplequantum-well perovskites for high-performance light-emitting diodes. Adv. Mater. 32(15), 1904163 (2020). https://doi.org/ 10.1002/adma.201904163

46. Z. Ren, K. Wang, X.W. Sun, W.C.H. Choy, Strategies toward efficient blue perovskite light-emitting diodes. Adv. Funct. Mater. 31(30), 2100516 (2021). https://doi.org/10.1002/adfm. 202100516

47. S. Yang, J. Dai, Z. Yu, Y. Shao, Y. Zhou et al., Tailoring passivation molecular structures for extremely small opencircuit voltage loss in perovskite solar cells. J. Am. Chem. Soc. 141(14), 5781-5787 (2019). https://doi.org/10.1021/jacs. $8 b 13091$

48. Z. Chu, Q. Ye, Y. Zhao, F. Ma, Z. Yin et al., Perovskite lightemitting diodes with external quantum efficiency exceeding $22 \%$ via small-molecule passivation. Adv. Mater. 33(18), 2007169 (2021). https://doi.org/10.1002/adma.202007169

49. W. Xu, Q. Hu, S. Bai, C. Bao, Y. Miao et al., Rational molecular passivation for high-performance perovskite light-emitting diodes. Nat. Photon. 13, 418-424 (2019). https://doi.org/10. 1038/s41566-019-0390-х

50. J. Yao, L. Wang, K. Wang, Y. Yin, J. Yang et al., Calciumtributylphosphine oxide passivation enables the efficiency of pure-blue perovskite light-emitting diode up to $3.3 \%$. Sci. Bull. 65(14), 1150-1153 (2020). https://doi.org/10.1016/j. scib.2020.03.036

51. D.W. deQuilettes, S. Koch, S. Burke, R.K. Paranji, A.J. Shropshire et al., Photoluminescence lifetimes exceeding $8 \mu$ s and quantum yields exceeding $30 \%$ in hybrid perovskite thin films by ligand passivation. ACS Energy Lett. 1(2), 438-444 (2016). https://doi.org/10.1021/acsenergylett.6b00236

52. X. Yang, X. Zhang, J. Deng, Z. Chu, Q. Jiang et al., Efficient green light-emitting diodes based on quasi-two-dimensional composition and phase engineered perovskite with surface passivation. Nat. Commun. 9, 570 (2018). https://doi.org/10. 1038/s41467-018-02978-7

53. D.P. McMeekin, Z. Wang, W. Rehman, F. Pulvirenti, J.B. Patel et al., Crystallization kinetics and morphology control of formamidinium-cesium mixed-cation lead mixed-halide perovskite via tunability of the colloidal precursor solution. Adv. Mater. 29(29), 1607039 (2017). https://doi.org/10.1002/ adma.201607039

54. Y. Shang, Y. Liao, Q. Wei, Z. Wang, B. Xiang et al., Highly stable hybrid perovskite light-emitting diodes based on DionJacobson structure. Sci. Adv. 5(8), eaaw8072 (2019). https:// doi.org/10.1126/sciadv.aaw8072

55. Z. Chen, C. Zhang, X.F. Jiang, M. Liu, R. Xia et al., Highperformance color-tunable perovskite light emitting devices through structural modulation from bulk to layered film. Adv. Mater. 29(8), 1603157 (2017). https://doi.org/10.1002/adma. 201603157

56. Z. Ren, L. Li, J. Yu, R. Ma, X. Xiao et al., Simultaneous loworder phase suppression and defect passivation for efficient and stable blue light-emitting diodes. ACS Energy Lett. 5(8), 2569-2579 (2020). https://doi.org/10.1021/acsenergylett. 0c01015

57. F. Zhang, B. Cai, J. Song, B. Han, B. Zhang et al., Efficient blue perovskite light-emitting diodes boosted by 2D/3D energy cascade channels. Adv. Funct. Mater. 30(27), 2001732 (2020). https://doi.org/10.1002/adfm.202001732

58. Y. Yao, H. Yu, Y. Wu, Y. Lu, Z. Liu et al., Efficient quantum dot light-emitting diodes based on trioctylphosphine oxidepassivated organometallic halide perovskites. ACS Omega 4(5), 9150-9159 (2019). https://doi.org/10.1021/acsomega. $9 \mathrm{~b} 00464$

59. L. Xie, J. Chen, P. Vashishtha, X. Zhao, G.S. Shin et al., Importance of functional groups in cross-linking methoxysilane additives for high-efficiency and stable perovskite solar cells. ACS Energy Lett. 4(9), 2192-2200 (2019). https://doi. org/10.1021/acsenergylett.9b01356

60. D. Bi, C. Yi, J. Luo, J.D. Décoppet, F. Zhang et al., Polymertemplated nucleation and crystal growth of perovskite films for solar cells with efficiency greater than $21 \%$. Nat. Energy 1, 16142 (2016). https://doi.org/10.1038/nenergy.2016.142

61. L. Zhang, X. Yang, Q. Jiang, P. Wang, Z. Yin et al., Ultrabright and highly efficient inorganic based perovskite lightemitting diodes. Nat. Commun. 8, 15640 (2017). https://doi. org/10.1038/ncomms 15640

62. C. Katan, N. Mercier, J. Even, Quantum and dielectric confinement effects in lower-dimensional hybrid perovskite semiconductors. Chem. Rev. 119(5), 3140-3192 (2019). https://doi. org/10.1021/acs.chemrev.8b00417

63. J. Liu, J. Leng, K. Wu, J. Zhang, S. Jin, Observation of internal photoinduced electron and hole separation in hybrid twodimentional perovskite films. J. Am. Chem. Soc. 139(4), 1432-1435 (2017). https://doi.org/10.1021/jacs.6b12581

64. X. Qu, N. Zhang, R. Cai, B. Kang, S. Chen et al., Improving blue quantum dot light-emitting diodes by a lithium fluoride interfacial layer. Appl. Phys. Lett. 114, 071101 (2019). https:// doi.org/10.1063/1.5087102

65. F. Yang, H. Chen, R. Zhang, X. Liu, W. Zhang et al., Efficient and spectrally stable blue perovskite light-emitting diodes based on potassium passivated nanocrystals. Adv. Funct. Mater. 30(10), 1908760 (2020). https://doi.org/10.1002/adfm. 201908760

66. Y. Jin, Z.K. Wang, S. Yuan, Q. Wang, C. Qin et al., Synergistic effect of dual ligands on stable blue quasi-2D perovskite lightemitting diodes. Adv. Funct. Mater. 30(6), 1908339 (2020). https://doi.org/10.1002/adfm.201908339

67. Y. Shen, K.C. Shen, Y.Q. Li, M. Guo, J. Wang et al., Interfacial potassium-guided grain growth for efficient deep-blue perovskite light-emitting diodes. Adv. Funct. Mater. 31(6), 2006736 (2020). https://doi.org/10.1002/adfm.202006736

68. M. Worku, Q. He, L. Xu, J. Hong, R.X. Yang et al., Phase control and in situ passivation of quasi-2D metal halide perovskites for spectrally stable blue light-emitting diodes. ACS Appl. Mater. Interfaces 12(40), 45056-45063 (2020). https:// doi.org/10.1021/acsami.0c12451 
69. N. Yantara, N.F. Jamaludin, B. Febriansyah, D. Giovanni, A. Bruno et al., Designing the perovskite structural landscape for efficient blue emission. ACS Energy Lett. 5(5), 1593-1600 (2020). https://doi.org/10.1021/acsenergylett.0c00559

70. F. Yuan, C. Ran, L. Zhang, H. Dong, B. Jiao et al., A cocktail of multiple cations in inorganic halide perovskite toward efficient and highly stable blue light-emitting diodes. ACS Energy
Lett. 5(4), 1062-1069 (2020). https://doi.org/10.1021/acsen ergylett.9b02562

71. D. Ma et al., Chloride insertion-immobilization enables bright, narrowband, and stable blue-emitting perovskite diodes. J. Am. Chem. Soc. 142(11), 5126-5134 (2020). https://doi.org/10.1021/jacs.9b12323 